\title{
Cultura de seguridad de pacientes en profesionales de la salud en instituciones hospitalarias de Río Gallegos: Cultura de seguridad del adulto mayor
}

\author{
Pacientepatient safety culture in health professionals in Río Gallegos hospital \\ institutions: Security culture of the adult major
}

\author{
Gabriela Bettina Perez \\ bettinaperez2016@gmail.com \\ Escuela de Salud Pública- UNC \\ Universidad Nacional de la Patagonia Austral, Río Gallegos, Santa Cruz \\ ISISC- UARG \\ Argentina
}

Recibido: 04/12/2019. Aceptado: 11/09/2020

\begin{abstract}
RESUMEN
Dado el progresivo envejecimiento poblacional, los sistemas sanitarios deberán implementar políticas para una atención segura y de calidad. La propuesta de este trabajo es reflexionar y analizar la seguridad del paciente a través de los profesionales de la salud en dos Hospitales de la ciudad de Río Gallegos, Santa Cruz. Se presenta un diseño metodológico para la aplicación de un instrumento ya validado, versión traducida al español del cuestionario Hospital Surveyon Patient safety culture.
\end{abstract}

Palabras clave: seguridad en paciente; cultura organizacional; gestión de seguridad; adulto mayor.

\begin{abstract}
Due to the progressive aging of the population, health systems require the implementation of policies for safe and quality care. The aim of this study is to reflect and analyze patient safety through health professionals in two Hospitals of the city of Río Gallegos, Sta Cruz. A methodological design for the application of an already validated instrument is presented, a version translated into Spanish of the questionnaire Hospital Surveyvon Patient safety culture.
\end{abstract}

Keywords: patient safety; organizational culture; safety management; elderly.

\section{INTRODUCCIÓN}

La cultura de seguridad del paciente se concibe (Weaver, 2013) como la suma de valores, actitudes, percepciones, competencias y patrones de conducta individual y grupal; que determinan el compromiso en la gestión de la seguridad en la atención en el cuidado del paciente y garantizan que no experimentará daño innecesario o potencial asociado a la atención en salud. La cultura de seguridad puede influir en los procesos de atención y sus 
resultados, es así que los esfuerzos para evaluar el clima de seguridad del paciente a lo largo del tiempo se están implementando ampliamente (Halligan, 2011).

Una cultura positiva sobre seguridad del paciente en las instituciones sanitarias se perfila como uno de los requisitos esenciales para evitar, en lo posible, la aparición de efectos adversos, y poder aprender de los errores de forma proactiva, para rediseñar los procesos de manera que los errores no se vuelvan a producir (García Casanova, 2014).

Un evento adverso (EA) es un incidente que ocasiona un daño innecesario a un paciente (Mira, 2013). Estudios realizados sobre eventos adversos, en Argentina, revelan que los informes deben permitir identificar los eventos que conducen a los incidentes para abordar intervenciones efectivas en seguridad de pacientes, involucrando todo el personal, con énfasis en el personal directivo (Ramos, Abeldaño, 2104).

El movimiento de seguridad del paciente tuvo su inicio en la última década del siglo XX, tras la publicación del Informe (To Erris Human) quien presentó resultados de varios estudios que revelaron datos sorprendentes, comparando la cantidad de muertes en los Estados Unidos, con la caída de un jumbo por día (Pinheiro, 2017).

En la Asamblea Mundial de la Salud del año 2002, los Estados Miembros de la Organización Mundial de la Salud (OMS) adoptan una resolución sobre la seguridad del paciente. Actualmente se reconoce cada vez más que la seguridad del paciente y la calidad de la atención que se le presta son dimensiones fundamentales de la Cobertura Sanitaria Universal. Cada año, cientos de miles de pacientes en todo el mundo son lesionados o mueren mientras reciben asistencia sanitaria. Los datos de la OMS y otras fuentes indican que los incidentes contra la seguridad del paciente se producen entre un $4 \%$ y un $16 \%$ de las personas hospitalizadas. Debido a los complejos procesos asistenciales tecnológicos y humanos, la OMS propone la "Alianza Mundial para la Seguridad del Paciente" en el año 2004, con el propósito de coordinar, difundir y acelerar la mejora en materia de seguridad de paciente en todo el mundo.

La primera estrategia para mejorar la seguridad del paciente pasa por promover, crear y mantener una cultura de la seguridad positiva en las instituciones (García-Barbero, 2005). El trabajo en equipo, el soporte de los líderes y la comunicación son atributos que contribuyen a la seguridad del paciente (Nieva, 2004).

La población de las Américas ha ganado 16 años de vida como promedio en los últimos 45 años, Ahora una persona que nace en el continente puede aspirar a vivir hasta los 75 años, casi 5 años más que el promedio mundial. Sin embargo, las enfermedades emergentes y las no transmisibles, que causan cuatro de cada cinco muertes anuales. (OPS/OMS, 2017) Proyecciones en base a resultados del Censo Nacional de población INDEC, en la provincia de Santa Cruz, la esperanza de vida será de 71,47 para los hombres y 79,43 años para las mujeres. La población de adultos mayores de más de 65 años, representa el $6 \%$ de la población total de santa cruz- (INDEC -2017)

El envejecimiento es un fenómeno presente a lo largo del ciclo vital desde el mismo proceso de la concepción hasta la muerte. Actualmente se considera un problema de salud pública, ya que el cambio en la pirámide poblacional se ha volcado hacia el aumento de esta población (García-Maya, 2014). 
En esta investigación de la cultura de seguridad de pacientes en profesionales de la salud en instituciones hospitalarias en Rio Gallegos, se realiza una mirada hacia el adulto mayor sobre sus cuidados, hospitalización y trabajo en equipo. El trabajo en este tema de seguridad es de vital importancia, tal como lo es buscar evidencia que promueva el análisis en torno a la seguridad de los pacientes adultos mayores, aportando seguridad en los profesionales que estén más capacitados, produciendo mejores resultados en el cuidado de los pacientes, y simultáneamente incrementando la calidad y confianza de las instituciones de salud.

El objetivo de este estudio es analizar la cultura de seguridad de los pacientes, a través de los profesionales de la salud, en las dos Instituciones Hospitalarias de Río Gallegos, Provincia de Santa Cruz.

\section{Adulto mayor hospitalizado}

Cabe señalar que la Organización Mundial de la Salud (OMS) considera como adulto mayor a toda persona mayor de 60 años.

Cuidar -esencia de la profesión de enfermería- implica establecer una relación de ayuda con el paciente y su familia, para lo cual es necesaria la relación estrecha y comunicación efectiva. Dado el aumento en la expectativa de vida y consecuente incremento de la población adulta mayor, aparece una mayor exigencia sobre el recurso humano, se requiere entrenamiento e intuición para la toma de decisiones al realizar acciones o actividades que respondan a las necesidades particulares de los pacientes de este grupo de edad. (Chávez- Cáceres, 2018).

Evidencias científicas así como la experiencia profesional demuestran que la hospitalización supone para el anciano factores de riesgos, independientemente de la causa del ingreso. La OMS, 1974, define factores de riesgo "al período inmediato posterior al alta hospitalaria". Desde el punto de vista geriátrico el riesgo se entiende como la probabilidad que tienen los ancianos de ser incapaces de enfrentarse a las necesidades vitales. Así mismo, la hospitalización facilita la aparición de complicaciones, sin relación con la enfermedad base, como el aumento del riesgo de úlceras por presión, vinculado con el estado de la piel y de la nutrición, la facilidad para las caídas, con unas consecuencias físicas, psíquicas y socioeconómicas que las hacen ser una de las principales causas de morbimortalidad en los ancianos otros factores de riesgo que deben valorarse se encuentran en los antecedentes de caídas anteriores. Además pueden presentarse neumonías u otras infecciones, incontinencia fecal y/o urinaria, trombo embolismo, que aumentan el deterioro psicofuncional del anciano (Canga Armayor, 2006).

La seguridad del paciente pretende evitar los incidentes y eventos adversos durante la atención en salud, ya que los pacientes en estos procesos, tienen un alto grado de vulnerabilidad, consecuentemente las instituciones debemos trabajar en pro de una atención segura, al recibir la atención deseada, lo que conlleva a una estancia menor.

\section{Trabajar en equipo de salud}

Trabajar todos como equipo de una institución en crear una cultura fuerte de seguridad del paciente, con el fin de incentivar el reporte de los incidentes y eventos adversos para lograr mejoras y evitar que se vuelvan a presentar, esto debe ir acompañado de una constante capacitación en seguridad del paciente, el manejo de medicamentos y dispositivos médicos, venopunción, caídas, manejo de pacientes adultos con estancias prolongadas para evitar las ulceras, correcto uso de las medidas de bioseguridad, infecciones intrahospitalarias de la mano de una campaña de lavado de manos, incentivar el registro en la historia clínica de los 
eventos adversos y sobre todo de un gran apoyo por parte de la administración y/o Gerencia de la institución (Prado Cuellar, 2014).

El resultado de nuevas investigaciones sobre el tema de seguridad en pacientes, busca esclarecer lo que sucede en la práctica profesional y así proponer estrategias para evitar la repetición de errores mejorando la cultura organizacional y con eso, la prevención de eventos adversos y la mejoría de la calidad y seguridad de la atención al paciente (Camargo Tobías, 2014).

\section{Autocuidado, educación, familia}

El cuidado es una función cotidiana que empleamos para sobrellevar un determinado nivel de vida, cuidar representa un conjunto de actos de vida que tiene por objetivo hacer que ésta se mantenga, continúe y se desarrolle.

Al mismo tiempo se está viendo que la proporción de enfermos ancianos que ocupan camas hospitalarias es cada vez mayor, de ahí la importancia de una valoración integral que, sin sustituir la historia clínica habitual, determine los riesgos de deterioro funcional y de aparición de complicaciones para poder planificar cuidados específicos para la población anciana hospitalizada que minimicen o prevengan los principales problemas que puedan presentar.

El profesional de enfermería en el desempeño de su labor proporciona cuidados a la salud, así como la enseñanza del autocuidado del usuario que aplicará en su vida cotidiana, fuera de alguna institución de salud y esta respaldara un óptimo estado de salud.

Así mismo se considera de gran importancia aplicar cuidados de educación, al anciano y acompañante, durante la hospitalización para fomentar el autocuidado durante el ingreso y al alta (Canga Armayor, 2016).

El autocuidado comprende todas las acciones y decisiones que toma una persona para prevenir, diagnosticar y tratar su enfermedad, todas las actividades individuales dirigidas a mantener y mejorar la salud, y las decisiones de utilizar tanto los sistemas de apoyo formales de salud como los informales. Dorothea Orem, define en su teoría al autocuidado como "la práctica de actividades que una persona inicia y realiza por su propia voluntad para mantener la vida, la salud y el bienestar". Afirma, que el autocuidado es una conducta que se aprende y surge de combinaciones de experiencias cognitivas y sociales. El autocuidado se convierte en un elemento fundamental de promoción y determinación en la calidad de vida ya que permite al ser humano ejercer su autonomía, decidir sobre su propio bienestar y participar activamente en acciones que mejoren las condiciones en que se desenvuelve (Peña,2011).

Existen evidencias de que cuando el paciente pregunta, se informa, participa de su cuidado y del restablecimiento de su salud, adopta un papel más activo, mejoran los resultados clínicos y se incrementa la seguridad, al tiempo que disminuye la probabilidad de error (Aranaz,2004).

\section{Metodología}

El estudio de alcance descriptivo, de corte transversal realizado durante los meses de agosto y septiembre del año 2019 en la ciudad de Río Gallegos, en las siguientes instituciones hospitalarias: Hospital Regional Río gallegos y Hospital Militar de Río Gallegos. La 
Población de estudio fue conformada por profesionales de la salud; médicos, residentes, farmacéuticos, licenciados, técnicos y auxiliares, que estuvieran en contacto directo con el paciente. La población de estudio compuesta por 400 profesionales en las dos instituciones, quedó constituida por una muestra de 193 profesionales que aceptaron participar en el estudio, lo que hace una tasa de respuesta de 48,25\%. El criterio de inclusión exigió que haya tenido una antigüedad mínima de seis (6) meses en la institución.

El instrumento para la recolección de datos, es un cuestionario individual auto- administrado (Agencia para la Investigación, calidad, 2005) validado como instrumento fiable para medir actitudes y comportamientos relacionados con la seguridad del paciente en los profesionales, ya que permite obtener información acerca de la cultura de seguridad y posteriormente indicar fortalezas y debilidades. Los profesionales que integraron la muestra se le aplico el instrumento Hospital Surveyon Patient safety culture, adaptado por el grupo de investigación de la Universidad de Murcia. A la encuesta se le adjuntó el consentimiento, el cual fue voluntario y anónimo aceptando formar parte del estudio.

\section{Diseño estadístico}

El tratamiento estadístico de estos datos se está realizando con medidas de tendencia central y de dispersión y estadísticos bivariados utilizando el paquete SPSS versión 18 con una significación de $\mathrm{p}<0,05$.

Con metodología cuantitativa, en la primera fase el análisis de los datos es descriptivo por variable de estudio, ya que se referirá a los hechos como son observados, sin manipulación de variables.

En el análisis inferencial, el análisis de asociaciones entre variables categóricas, se utiliza el test de chi cuadrado y el test de student para las variables cuantitativas.

La determinación de confiabilidad y validez del instrumento se aplicará el coeficiente alfacronbach. (Coeficientes que oscilan entre 0 y 1 , donde 0 representa nula confiabilidad y 1 máxima confiabilidad).

Para el grado de seguridad del paciente, se utiliza el coeficiente de relación por rangos de Spearman (rs) para el nivel de medición ordinal.

\section{Resultados}

La muestra quedó integrada por 193 profesionales, que se desempeñaban en distintas áreas de las instituciones. El personal de enfermería representó el 36,8\% del total de los profesionales que accedieron al estudio, siendo éstos los de mayor aceptación a la propuesta de investigación. En la tabla 1, de la distribución, muestra la participación de los trabajadores de salud en sus distintos puestos de trabajo, en este caso enfermería quien tienen relación directa con los pacientes y su cuidado a cargo, da cuenta la importancia de la cultura de seguridad en el fortalecimiento dentro de la organización del hospital, identificando áreas y oportunidad de mejora continua, para proporcionar cuidados de calidad en este caso al adulto mayor. 
Tabla 1 Distribución del puesto de trabajo actual.

\begin{tabular}{|c|c|c|}
\hline Profesión & Frecuencia & Porcentaje \\
\hline & 1 & 0,5 \\
\hline Enfermero/a & 71 & 36,8 \\
\hline Obstetra & 3 & 1,6 \\
\hline Residente & 2 & 1,0 \\
\hline Médico & 25 & 13,0 \\
\hline Otros & 43 & 22,3 \\
\hline Anestesiólogo & 1 & 0,5 \\
\hline Farmacéutico & 3 & 1,6 \\
\hline Fisioterapeuta & 1 & 0,5 \\
\hline Fonoaudiólogo & 4 & 2,1 \\
\hline $\begin{array}{l}\text { Instrumentadores } \\
\text { Quirúrgicos }\end{array}$ & 4 & 2,1 \\
\hline Kinesiólogo & 3 & 1,6 \\
\hline Musicoterapeuta & 1 & 0,5 \\
\hline Odontólogo & 7 & 3,6 \\
\hline Psicólogo & 4 & 2,1 \\
\hline Psicopedagoga & 1 & 0,5 \\
\hline Radiólogo & 4 & 2,1 \\
\hline Técnico & 11 & 5,7 \\
\hline Trabajosocial & 4 & 2,1 \\
\hline Total & 193 & 100,0 \\
\hline
\end{tabular}

Fuente: Encuesta aplicada

Tabla 2. Distribución de trabajo en equipo

\begin{tabular}{|l|l|l|l|}
\hline & $\begin{array}{l}\text { Muy en desacuerdo/En } \\
\text { desacuerdo }\end{array}$ & Indiferente & $\begin{array}{l}\text { De acuerdo/Muy de } \\
\text { acuerdo }\end{array}$ \\
\hline $\begin{array}{l}\text { El personal se apoya } \\
\text { mutuamente }\end{array}$ & $22,3 \%$ & $10,9 \%$ & $66,9 \%$ \\
\hline $\begin{array}{l}\text { Cuando tenemos mucho } \\
\text { trabajo, colaboramos } \\
\text { todos como equipo para } \\
\text { poder terminarlo }\end{array}$ & $17,4 \%$ & $13,7 \%$ & $45,82 \%$ \\
\hline $\begin{array}{l}\text { En esta unidad nos } \\
\text { tratamos todos con } \\
\text { respeto }\end{array}$ & $21,4 \%$ & $13 \%$ & $65,6 \%$ \\
\hline $\begin{array}{l}\text { Cuando alguien está } \\
\text { sobrecargado de trabajo, } \\
\text { suele encontrar ayuda en } \\
\text { los compañeros }\end{array}$ & $16 \%$ & $17,1 \%$ & $66,8 \%$ \\
\hline
\end{tabular}

Fuente: encuesta aplicada 
En trabajo de equipo, en la tabla 2, los ítems con mayor porcentaje de frecuencia fueron: el personal se apoya mutuamente, en esta unidad nos tratamos todos con respeto, cuando alguien está sobrecargado de trabajo, suele encontrar ayuda en los compañeros. Esto evidencia que hay una tendencia o voluntad de los profesionales a realizar todo lo posible como equipos de trabajo para brindar lo mejor a los pacientes.

Estos son algunos de los resultados parciales de la tesis, la propuesta y los objetivos están orientados a identificar en su análisis las fortalezas y oportunidades de mejora que permitirán a estas instituciones diseñar estrategias, como así también propuestas de intervención, tratar la seguridad del paciente como un tema prioritario en los equipos de salud.

\section{Conclusión}

Se ha demostrado que tener en cuenta la seguridad del paciente en las instituciones de salud, crea una cultura de seguridad en todos los profesionales, incentivando el reporte de los incidentes y eventos adversos con el fin de analizarlos e implementar acciones de mejora. Trabajar en la identificación para la prevención, involucrando en el proceso de atención al paciente, a su familia, la comunicación para la oportuna, efectiva y de calidad en la atención (Prado Cuellar, 2014).

La seguridad del paciente convertida en la principal política en las organizaciones de salud, permite la implementación de estrategias y planes de seguimiento en pro de garantizar una atención segura, de ahí el compromiso que tenemos para promover una cultura que se traduzca en beneficio para todos.

En este marco el envejecimiento, representa un reto para la sociedad y los sistemas de salud.

\section{Sugerencias}

Se recomienda para todas las instituciones que prestan servicios de salud crear un sistema de reporte de incidentes y eventos adversos, identificando las causas, realizar un correcto análisis e implementar acciones de mejoramiento o barreras, con el fin de no repetir los mismos errores. Las mejores instituciones son las que detectan los problemas y hacen los estudios para solucionarlos e implementar barreras.

En consideración al cuidado de enfermería, autores como: Contreras, Coronado, López; 2008, recomiendan realizar estudios sobre el cuidado que se brinda; en particular con el grupo de población que mayor atención requiere: los adultos mayores. Como así también generar conocimiento a partir de la experiencia de los receptores del cuidado, conocimiento que sea útil para planear mejor las acciones de enfermería; y de esta manera, lograr la eficiencia en el cuidado de enfermería en este vulnerable grupo de población, como son los adultos mayores.

Como investigadora en este estudio, resalto la importancia de la seguridad del paciente en los distintos servicios y ámbitos hospitalarios, creando conciencia de cultura en seguridad, esencial para cuidar y atender en forma segura a los pacientes y así poder evitar posibles errores y brindar cuidados de calidad. 


\section{Referencias Bibliográficas}

AGENCIA PARA LA INVESTIGACIÓN, CALIDAD. Cuestionario sobre seguridad de los pacientes. Madrid: Ministerio de Sanidad y Consumo; 2005. http://www.msc.es/ organizacion/sns/planCalidadSNS/docs/CuestionarioSeguridadPacientes1.pdf

ALIANZA MUNDIAL PARA LA SEGURIDAD DEL PACIENTE. Disponible: www.who.int/patientsafety/information_centre/.../ps_research_brochure_es.pdf

ALVARADO GARCÍA A. M., MAYA S., MARÍA, Á. (2014). Análisis del concepto de envejecimiento. Gerokomos, 25(2), 57-62.

ARANAZ J. M., VITALLER J., GRUPO DE ESTUDIO DEL PROYECTO IDEA. (2004). De las complicaciones y efectos adversos a la gestión de los riesgos de la asistencia sanitaria. Estudios para la salud, (13).

CAMARGO TOBÍAS G., QUEIROZ BEZERRA A. L., SILVESTRE BRANQUINHO N. C., \& DE CAMARGO SILVA A. E. B. (2014). Cultura de la seguridad del paciente en la atención sanitaria: un estudio bibliométrico. Enfermeria global, 13(33), 336-348.

CANGA ARMAYOR A. D., NARVAIZA SOLÍS M. J. (2006). Intervención de enfermería para disminuir las complicaciones derivadas de la hospitalización en los ancianos. Gerokomos, 17(1), 24-31

CONTRERAS NUÑEZ R., CORONADO MARTÍNEZ M., LÓPEZ VILLANUEVA N., AGUILAR HERNÁNDEZ R. M., FÉLIX ALEMÁN A., VEGA ALANÍS M. D. C (2008). Satisfacción con el cuidado de Enfermería del adulto mayor hospitalizado. http://www.w3.org/1999/xhtml. [Fecha de Consulta 6 de Octubre de 2019]. ISSN: 1665-7063. Disponible en: https://www.redalyc.org/articulo.oa?id=3587/358741824 $\underline{003}$

CHÁVEZ CÁCERES A. (2018). Nivel de satisfacción del paciente adulto mayor respecto a los cuidados de enfermería en el servicio de medicina del hospital II- Minsa Tarapoto, año 2017.

DA PENHAPINHEIRO M., DA SILVA JUNIOR O. C. (2017). Avaliação da cultura de segurança do paciente naorganizaçãohospitalar de um hospital universitário. Enfermería Global, 16(1), 309-352.

GARCÍA-BARBERO M. (2004). La alianza mundial para la seguridad del paciente. Monografías humanitas, 8, 209-220.

GARCÍA CASANOVA Y., NADER DÍAZ S., MARULANDA PÉREZ A. (2014). La seguridad del paciente como paradigma de la excelencia del cuidado en los servicios de salud. Revista Cubana de Enfermería, 30(1), 62-64. Recuperado en 31 de octubre de 2019, de http://scielo.sld.cu/scielo.php?script=sci_arttext\&pid=S0864-031920 $14000100007 \& \operatorname{lng}=\mathrm{es} \& \operatorname{tlng}=\mathrm{es}$

HALLIGAN M., ZECEVIC A. (2011). Safety culture in healthcare: a review of concepts, dimensions, measures and progress. BMJ quality\& safety, 20(4), 338-343.

INDICADORES BÁSICOS-2019. http://www.deis.msal.gov.ar/wp-content/uploads/ 2019/12/Indicadores-Basicos-2019.pdf

MIRA J. J., CHO M., MONTSERRAT D., RODRÍGUEZ J., SANTACRUZ J. (2013). Elementos claves en la implantación de sistemas de notificación de eventos adversos hospitalarios en América Latina. Revista Panamericana de Salud Pública, 33, 01-07. 
OMS | 10 datos sobre seguridad del paciente. Asamblea Mundial de la Salud.2002. www.who.int/features/factfiles/patient_safety/patient_safety_facts/es/

ORGANIZACIÓN PANAMERICANA DE SALUD. https://www.paho.org/hq/index. php?option=com_content $\&$ view $=$ article $\&$ id $=13722$ :life-expectancy-in-the-americasincreases-to-75-years \&Itemid=1926\&lang=es

PEÑA J. V., ENRIQUE L. (2011) Educación como estrategia para lograr el autocuidado en.

PRADO CUELLAR M. C., RIZO RUIZ A. V., DÍAZ DÍAZ P. F., HOYOS RODRÍGUEZ A. E. (2014). Seguridad del paciente un compromiso institucional para una atención más segura.

RAMOS F., COCA S. M., ABELDAÑO R. A. (2017). Percepción de la cultura de seguridad de pacientes en profesionales de una institución argentina. Enfermería Universitaria.2017.jan:14(1):47-53. Disponible en http://linkinghub.elsevier.com/ retrieve/pii/S1665706316300720

SORRA J. S., NIEVA V. F. (2004). Hospital Survey on Patient Safety Culture (Agency for Healthcare Research and Quality Publication No. 04-0041). Rockville, MD: Agency for Healthcare Research and Quality.

WEAVER S. J., LUBOMKSI L. H., WILSON R. F., PFOH E. R., MARTINEZ K. A., DY S. M. (2013). Promoción de una cultura de seguridad como estrategia de seguridad del paciente: una revisión sistemática. Anales de medicina interna, 158 (5_Parte_2), 369374. 